\title{
Multiple-period dispersion-managed solitons
}

\author{
O. Y. Schwartz ${ }^{1}$ and S. K. Turitsyn ${ }^{2}$ \\ ${ }^{1}$ Weizmann Institute of Science, Rehovot, 76100, Israel \\ ${ }^{2}$ Photonics Research Group, Aston University, Birmingham B4 7ET, United Kingdom
}

(Received 30 March 2007; published 15 October 2007)

\begin{abstract}
We present a class of solutions with a period multiple to that one of the standard dispersion-managed soliton in the nonlinear Schrödinger equation with periodic variations of dispersion.

DOI: 10.1103/PhysRevA.76.043819

PACS number(s): 42.81.-i, 42.65.Tg
\end{abstract}

\section{INTRODUCTION}

Nonlinear systems with periodic variations of one or several key parameters present a very important branch of nonlinear science with a range of practical applications in solid state physics, optics, plasma physics, hydrodynamics, wave theory, and other research areas. Very often, nonlinear models governing rather different physical phenomena engaging nonlinearity and periodicity are quite similar mathematically. Therefore, analysis of certain generic nonlinear models might be interesting for a range of physical applications. One of the important examples of such systems is the nonlinear Schrödinger equation (NLSE) that is one of the key basic models of the nonlinear wave physics. It is used to describe high-frequency nonlinear wave propagation in medium with Kerr-type nonlinearity. This model is very generic because it is derived under two rather general assumptions: first, a high carrier frequency of the propagating wave envelope and a parabolic approximation of the dispersion curve near the carrier frequency, and second, the nonlinearity is assumed to be proportional to the intensity of the propagating wave field (Kerr-type nonlinearity). These are, indeed, rather general assumptions that are well justified in numerous physical applications. The NLSE has been derived in such different physical areas as plasmas, hydrodynamics, nonlinear optics, fiber optics, solid state physics, and many others (see, e.g., Refs. [1-9]).

It should be mentioned that the interest to the NLSE model has been recently enhanced in the context of application to the Bose-Einstein condensate dynamics. The dynamics of a gas of cooled atoms confined in a magnetic trap at very low temperatures can be described by an effective NLS equation for the condensate wave function introduced in this physical context by Gross and Pitaevskii [10]. Another very important application of the NLS equation is in fiber optics and, more specifically, in optical signal transmission along dispersion-managed links. In fiber optic communications, a periodic dispersion management-alternation of the fiber spans with positive and negative dispersion is an established technique to improve system performance.

Stable recurring dynamics of certain classes of carrier pulse in the periodic dispersion map under combined action of nonlinear effects and dispersion is an interesting example of the extension of the traditional soliton theory to the case of so-called dispersion-managed solitons (see, e.g., Refs. [9,11-13], and references therein). Efficient growth of the capacity of digital communication systems can be achieved by increase of the channel bit rate-the speed at which information bits are transmitted. Increasing the channel rate assumes the utilization of shorter time slots allocated for each information bit and, consequently, of shorter carrier pulses. The propagation of ultrashort pulses is then strongly affected by the fiber dispersion, which results in large temporal broadening of the carrier pulses. Because of the temporal broadening during propagation, the carrier pulse power spreads over many time slots and, consequently, the accumulated effect of the instantaneous fiber nonlinearity tends to get averaged out. Signal transmission using very short optical pulses, often referred to as the quasilinear regime $[14,15]$, became a mainstream of the modern development in optical fiber communications. This regime is, in some sense, opposite to soliton or DM soliton transmission, where fiber nonlinearity plays an important role in preserving the pulse shapes during propagation. Note that in the quasilinear regime, the in-line Kerr nonlinearity is often considered almost as a "negative" factor contributing to the destabilization and distortion of carrier pulses. Traditional DM solitons typically have large energies for such short pulse widths (or do not exist at all), and are not practical for ultra-high-speed transmission. In other terms, the Kerr nonlinearity cannot stabilize single pulses in the form of traditional DM solitons at large map strengths. It would be of interest to find periodic solutions resembling DM solitons, while having smaller energies. As we will show below this feature (lower energy for the same pulse width) can be achieved considering solutions with periods multiple to that one of dispersion variation.

The multiple-period optical solitons in nonlinear systems with periodically changing parameters were first found in a different context [16]. In that work the optical solitons in a passively mode-locked fiber laser were studied. Although the cubic-quintic complex Ginzburg-Landau equation describing this system has much in common with the NLSE, the physics behind it is completely different (in contrast to the NLSE, the system is saturable, and not conservative). Therefore, behavior of the multiple-periodic solutions of the NLSE (which turn out to be qualitatively different from those of the Ginzburg-Landau equation) has to be studied separately.

In this paper, we analyze the multiple-periodic solutions of the NLSE (with periodic oscillations of the dispersion). These solutions can be useful in high-speed transmission, especially, in a combination with modulation formats with periodic distribution of intensity in time (having pulse in each time slot), e.g., differential phase-shift-keying (DPSK) formats. In terms of practical applications, further studies with analysis of practical dispersion maps are required to 
demonstrate advantage of such solutions. We would like to point out that described below multiple-period solutions present somehow an important step in convergence of the DM soliton concept and widely used in practical quasilinear transmission regimes. Namely, multiple-period DM solitons experience larger broadening during propagation and, therefore, they much more closely mimic "quasilinear" propagation regimes compared to traditional DM solitons. Note also that quasilinear transmission regimes similar to the ones in high-bit-rate systems can be implemented even in existing systems operating at a lower bit rate. For instance, as it was pointed out in Ref. [17], a pseudo-linear, bit-overlapping transmission regime that is typically attributed to $40 \mathrm{Gbit} / \mathrm{s}$ systems can be advantageously applied at $10 \mathrm{Gbit} / \mathrm{s}$ rates using short carrier pulses and RZ-DPSK format. Therefore, by varying the pulse duty cycle at $20 \mathrm{Gbit} / \mathrm{s}$ channel rate it is possible to realize rather different transmission regimes, ranging from a pseudolinear regime to a dispersion-managed solitonlike one. Multiple-period DM solitons described below might present an interesting transmission regime converging quasilinear and DM soliton concepts. Another possible practical application of multiple-period solutions that we would like to mention is related to laser systems using cavity dispersion management [18]. In this work we examine general features of the NLSE, rather than any specific application; however, without loss of generality we will use terminology relevant to fiber optical applications.

\section{MASTER EQUATION AND NOTATIONS}

The optical signal propagation down a fiber line with varying dispersion is governed by the NLSE equation (see, e.g., Refs. [11,12] for more details):

$$
i \frac{\partial A}{\partial z}+d(z) \frac{\partial^{2} A}{\partial t^{2}}+\sigma(z)|A|^{2} A=0 .
$$

Here the function $A(Z, t)$ is related to the envelope of the electrical field $\Psi(z, t)$ through equation $\Psi(z, t)=A(Z, t)$ $\times \exp \left[\int_{0}^{z} G\left(z^{\prime}\right) d z^{\prime}\right]$, where $G(z)$ is the effective gain or loss function along the fiber line. In other words, using field $A$ instead of $\Psi$ the power variations due to loss and amplification are included into the function $\sigma(z)$. We consider here normalized equations and refer to Refs. [11,19] for details of normalization. The function $\sigma(z)$ corresponds to signal power variations due to fiber loss and either distributed or lumped optical amplification that we assume to be periodic along the line. In submarine systems, a typical scale of the periodic variations of gain often is much smaller than the period of dispersion variations. Therefore, often, for simplicity it is considered a model averaged over shorter oscillations of power leading to the effective condition of a piecewise constant $\sigma(z)$. To simplify the calculations we limit our analysis by the so-called "lossless" case $\sigma(z)=$ const. As was discussed in Refs. [11,19] this situation corresponds to the limit that is rather typical for installed undersea systems with $L \gg Z_{a} ; Z_{a}$ is the amplification distance. This model also is applied in other important practical cases such as, for instance, recently demonstrated distributed amplification with ultralow power excursion level [20] quasilossless transmission. Moreover, the results presented here are not specific for and not limited by the lossless case, and the situation is qualitatively the same for varying periodical $\sigma$ (with period being a rational commensurable of $L)$. The function $d(z$ $+L)=d(z)$ describes periodic variation of the dispersion along the line. In this paper we consider a two-step map with piecewise-constant $\sigma(z)$ and $d(z)$ :

$$
\begin{aligned}
& d(z)=d_{1}>0, \quad \sigma=\sigma_{1}, \quad 0<z<L_{1}, \\
& d(z)=d_{2}<0, \quad \sigma=\sigma_{2}, \quad L_{1}<z<L,
\end{aligned}
$$

$L_{1}, L_{2}$ are the lengths of two pieces of fiber $L_{1}+L_{2}=L$.

The traditional DM soliton [21-23] is a solution of Eq. (1) that restores its form periodically with the periodic $L$. In the linear limit the NLSE is reduced to the linear Schrödinger equation. For linear equations with periodically varying coefficients, the Bloch's theorem states the existence of the solutions that are periodic functions with the same period, multiplied by a complex exponent that determines the Bloch wave vector. We will refer to such solutions simply as eigenfunctions of the given equation. The dispersion-managed soliton can be considered as a nonlinear Bloch wave function or, in other terms, the DM soliton is a nonlinear eigenfunction of Eq. (1) with the period $L$ that exists in the case of small nonlinearity: $A(z, t)=\exp [i \lambda z] F(z, t), \quad F(z+L, t)$ $=F(z, t)$. It turns out that along with the standard solutions with the period $L$, NLSE (1) also possesses a class of eigenfunctions with multiple periods $L_{n}=n L$, where $n$ is a natural number.

The solutions of NLSE (1) can be obtained from the eigenfunctions of the linear Schrödinger equation by an appropriate iteration procedure (see, e.g., Ref. [19], and references therein). The convergence of this procedure is determined by the smallness of the nonlinear coefficient in Eq. (1). One of the important results concerning the DM soliton that is relevant for this work is that when variations of the normalized function $d(z)$ are large (the so-called large dispersion map strength - to be defined later) the DM solitons either do not exist or they have too large energy to be practically used [24].

The goal of this work is to present a family of periodic structures representing eigenfunctions of Eq. (1) with period $L_{n}=n L$, where $n$ is a natural number. In general, such solutions have energy reduced compared to corresponding standard DM solitons and, therefore, could be of potential interest for high-speed transmission regimes. It is well established that DM soliton dynamics can be well approximated by analysis of a reduced model [11-13,25-28]. Therefore, first we examine the multiple period solutions analyzing a simplified model that approximates periodic solutions of the NLSE through a system of two ODEs that we will refer to through the text as the $T M$ (width $T$-chirp $M$ ) system. We will call through the paper the solutions with $n=1$ simple period solutions (or DM soliton) and those with $n>1$ long-period (or multiple-period) solutions. The simple period solutions of the $T M$ system have been comprehensively discussed in the literature (see, e.g., Refs. [11-13,25-28], and references 
therein). To the best of our knowledge, the long-period solutions have not yet been studied systematically and even the problem of the existence of such solutions has not been addressed. The structure of this paper is as follows. In the next section we reintroduce the $T M$ model, review its properties, and discuss the area of applicability. Next we consider the $T M$ model in the case of a two-step dispersion map, and describe properties of multiple-period solutions. Finally, we discuss periodic solutions in the full NLSE model.

\section{REDUCED MODEL: TM SYSTEM}

In the limit when dispersion dominates nonlinearity, the solution of NLSE (1) experiences quasilinear oscillations; namely, the key parameters, such as width $(T)$ and chirp $(M)$, during the evolution follows periodic variations of the dispersion. Effects of nonlinearity and small average dispersion come into play on much longer scales compared to $L$. First, let us remove this rapid evolution by transforming Eq. (1) to a more convenient form, where fast and slow dynamics are separated. This can be done by applying the following substitution [29] to (1):

$$
A(z, t)=N \frac{f(x, z)}{\sqrt{T(z)}} \exp \left[i \frac{M(z)}{T(z)} t^{2}\right], \quad x=t / T(z) .
$$

Parameter $N$ is introduced to scale out signal power by imposing a normalization condition on the structural (responsible for the pulse shape) function $f$ :

$$
\int|f|^{2} d x=1
$$

The functions $T(z)$ and $M(z)$ are to be defined so that the equation for $f$ would take the simplest form possible. First, note that if we would define the evolution of $T$ and $M$ by

$$
\begin{gathered}
\frac{d T}{d z}=4 d(z) M, \\
\frac{d M}{d z}=\frac{d(z)}{T^{3}}
\end{gathered}
$$

(that corresponds to the linear evolution) we would get for the function $f(x, z)$

$$
i \frac{\partial f}{\partial z}+\frac{d}{T^{2}}\left[\frac{\partial^{2} f}{\partial x^{2}}-x^{2} f\right]+N^{2} \frac{\sigma(z)}{T}|f|^{2} f=0 .
$$

Equation (6) is an evolution equation of the quantum harmonic oscillator with an additional nonlinear term. The nonlinearity mixes different modes of the linear oscillator, inducing transitions from the ground state (which corresponds to a Gaussian pulse) to other modes. We can reduce the rate of these transitions by including an additional term (that accounts in some sense for nonlinear pulse evolution) into the second equation of Eq. (5):

$$
\frac{d T}{d z}=4 d(z) M,
$$

$$
\frac{d M}{d z}=\frac{d(z)}{T^{3}}-\frac{p \sigma(z) N^{2}}{T^{2}} .
$$

We refer in what follows to this system of equations as the $T M$ equations. Here $p$ is some constant with a value that will be defined below in a way that minimizes the rate of the transitions from the ground mode to the higher order modes. This idea has effectively been used in Refs. [11,25-29]. We present here a more formal way to introduce nonlinearity into the equation for $M$.

Applying Eq. (7) we obtain

$$
i \frac{\partial f}{\partial z}+\frac{d}{T^{2}}\left[\frac{\partial^{2} f}{\partial x^{2}}-x^{2} f\right]+N^{2} \frac{\sigma(z)}{T}\left[|f|^{2} f+p x^{2} f\right]=0 .
$$

In order to compute an eigenfunction of Eq. (1) we have to find a periodic solution of system (7) $T(z), M(z)$, substitute $T(z)$ in Eq. (8), find its eigenfunction, and, finally, substitute it into Eq. (3)

We will adjust $p$ to minimize the rate of transitions from the ground state to other modes due to the nonlinear term in Eq. (8). This rate is the norm of the projection of $\partial f / \partial z$ onto the subspace, orthogonal to the ground state. Thus we have to minimize the norm of the vector

$$
a=(1-|f\rangle\langle f|)\left[|f(x)|^{2}+p x^{2}\right]|f\rangle .
$$

The standard quantum mechanics notation is used here, in which expression $|f\rangle\langle f|$ denotes the projection operator. The norm of this vector is quadratic in $p$, and it is simple to find that it is minimal when

$$
p=\frac{\left\langle|f(x)|^{2}\right\rangle\left\langle x^{2}\right\rangle-\left\langle|f(x)|^{2} x^{2}\right\rangle}{\left\langle x^{4}\right\rangle-\left\langle x^{2}\right\rangle^{2}} .
$$

Here the angle brackets denote a standard quantum mechanic averaging over $f:\langle g\rangle=\int|f(x)|^{2} g(x) d x$. The idea of the procedure is by optimizing parameter $p$ (that is related to the nonlinear term in $T M$ equations) to make the approximation of the DM soliton solution of the NLSE as close as possible to the ground state of the linear operator, Gaussian-shaped pulse. Therefore, we choose a constant $p$ by calculating Eq. (10) on the ground mode of the harmonic oscillator $f_{0}$ $=\pi^{-1 / 4} e^{-1 / 2 x^{2}}$. In this case $p \simeq 0.2$, and the rate of transitions is suppressed by a factor of the order of $C \sim 1 / 10$ in comparison with the case $p=0$. In other words, using nonlinear $T M$ equations with a properly selected $p$ makes a ground state-a Gaussian-shaped pulse-as close as possible to a true DM soliton solution.

It is known [22] that the shape of the DM soliton in a broad interval of parameters is close to Gaussian, therefore its exact form can be found by the perturbation theory $[11,12,19]$. This procedure converges if the rate of the transitions from the ground mode induced by the nonlinear term remains small. The deviation of the true eigenfunction of NLSE $f$ and Gaussian approximation (ground-state approximation) is given by 


$$
\delta f=f-f_{0} \sim \frac{C Q}{\max [1, S]},
$$

where $Q$ is the dimensionless nonlinear coefficient $Q$ $=L \sigma N^{2} / T, C$ is the small numerical coefficient mentioned above, and $S$ is the map strength defined (as in Ref. [24]) by

$$
S=2 \frac{L_{1} d_{1}-L_{2} d_{2}}{\min \left[T_{\mathrm{FWHM}}^{2}\right]} .
$$

(The full width at half maximum $T_{\text {FWHM }}[35]$ is taken at its minimum along the dispersion map.) This value characterizes the dimensionless length of the section of fiber, or the intensity of the dispersion-induced evolution. If $S \gg 1$, the nonlinear term can be accounted for as an adiabatic perturbation. Thus, the assumption that the shape of the pulse is close to Gaussian remains self-consistent for the values of $Q$ up to the order of magnitude $Q \sim \max [1, S]$. Thus, under this assumption, the problem of calculation of the eigenfunctions of NLSE (1) is reduced to the problem of the periodic solutions of the $T M$ system (7).

Note that $T M$ system (7) can also be regarded as an approximate system of evolution equations for integral rootmean-square momenta of the pulse width $[11,30] T$ is related to

$$
T_{\text {int }} \equiv \sqrt{\frac{\int t^{2}|A|^{2} d t}{\int|A|^{2} d t}}
$$

and the chirp $M$ corresponds to $M_{\text {int }}$ defined as

$$
T_{\mathrm{int}} M_{\mathrm{int}} \equiv \frac{i}{4} \frac{\int t\left(A A_{t}^{*}-A^{*} A_{t}\right) d t}{\int|A|^{2} d t}
$$

The evolution of $T_{\text {int }}, M_{\text {int }}$ is governed by Eq. (7) in the assumption that the phase of $A$ is quadratic in $t$. The accuracy of this assumption is the same as of the assumption that the pulse shape remains Gaussian during the propagation: relative error has the same order of magnitude that nonlinear term multiplied by a small numerical coefficient $(C \simeq 1 / 10)$.

\section{THE PERIODIC SOLUTIONS OF THE TM EQUATIONS FOR THE TWO-STEP MAP}

Without loss of generality, let us consider the TM system (7) on a two-step map (2). It is characterized by six physical parameters: length of the first piece $L_{1}$, its dispersion coefficient $d_{1}>0$, its nonlinearity coefficient $c_{1}=p \sigma_{1} N^{2}$, and the same for the second piece of fiber: $L_{2}, d_{2}<0$, and $c_{2}$. The TM system (7) allows three independent scale transformations which convert every solution of the initial system into a solution of transformed system. Two of them are trivial rescalings of variable $z$ :

$$
\begin{aligned}
& c_{1} \rightarrow \lambda c_{1}, \quad d_{1} \rightarrow \lambda d_{1}, \quad L_{1} \rightarrow \lambda^{-1} L_{1}, \\
& c_{2} \rightarrow \lambda c_{2}, \quad d_{2} \rightarrow \lambda d_{2}, \quad L_{2} \rightarrow \lambda^{-1} L_{2} .
\end{aligned}
$$

The third one is less obvious [36]:

$$
T \rightarrow \lambda T, \quad M \rightarrow \lambda^{-1} M, \quad c_{1,2} \rightarrow \lambda^{-1} c_{1,2}, \quad L_{1,2} \rightarrow \lambda^{2} L_{1,2} .
$$

All these transformations satisfy the rule of transition from one type of fiber to another, that is, $T(z)$ and $M(z)$ are continuous functions. We can use these symmetry transforms to scale out three of the parameters characterizing the coefficients of Eq. (7). For example, we can use the first two transforms to set $d_{1}=1$ and $d_{2}=-1$, and then use the third one to fix the length of the section $L=1$. In this normalization (it is essentially the same as used in Ref. [24]), the map is characterized by the three dimensionless parameters $c_{1}, c_{2}$ and average normalized dispersion $\bar{d}=L_{1}-L_{2}$. The map strength (11) then takes the form $S=2 / T_{\mathrm{FWHM}}^{2}$. In this paper, we only consider the case of "equally nonlinear" fibers, that is, $c_{1} / d_{1}=c_{2} / d_{2}=c$.

Let us briefly recall basic properties of simple periodic solutions of the TM system. Let us consider the trajectory of such a solution on the $(T, M)$ plane, starting in the point of transition from anomalous fiber to regular. The trajectory starts in some point $T^{*}, M^{*}$ and goes to the point $T^{*},-M^{*}$, reaching it at the moment $z=L_{1}$. Then the transition to regular fiber occurs, and the solution returns back to the point $T^{*}$, $M^{*}$ and reaches it at the moment $L_{1}+L_{2}$. The solution is symmetric with respect to inversion of $z$ (and $M$ ), so the trajectory crosses the $T$ axis at the moments $L_{1} / 2$ and $L_{1}$ $+L_{2} / 2$. So the condition that the solution is periodic can be written as

$$
z_{+}\left(T^{*}, M^{*}\right)=L_{1} / 2, \quad z_{-}\left(T^{*}, M^{*}\right)=L_{2} / 2,
$$

where $z_{ \pm}(T, M)$ is the length of anomalous or regular fiber that takes the solution to pass from the point with $M=0$ to a given point $T^{*}, M^{*}$. This function can be found analytically $[19,31]$ :

$$
z_{ \pm}=\frac{1}{2 V}\left[T M \mp \frac{c}{\sqrt{8 V}} \operatorname{arccosh}\left(\frac{T \pm c / 2 V}{\sqrt{c^{2} / 4 V^{2}+1 / 2 V}}\right)\right],
$$

where $V=\operatorname{const}(z)=2 M^{2}+0.5 / T^{2} \mp c / T$. Equations (16) provide a straightforward way to find simple periodic solutions of the TM system numerically. These solutions for some basic configurations are comprehensively described in the literature $[19,31]$. Note that often the application of these solutions in the field of optical data transmission is directly related to the energy dependence of $T_{\min } \equiv \min [T(z)]$. As the carrier pulse width is limited by the channel bit rate, the energy of the pulses should not be too high in real world units. The energy dependence is presented with thin lines in Fig. 1.

As it should be, $T_{\min }$ tends to a finite limit at $c \rightarrow 0$ in the case of zero $\bar{d}$, and tends to infinity for $\bar{d}>0$. For negative values of $\bar{d}$ the energy dependence of $T_{\min }$ has two branches. 


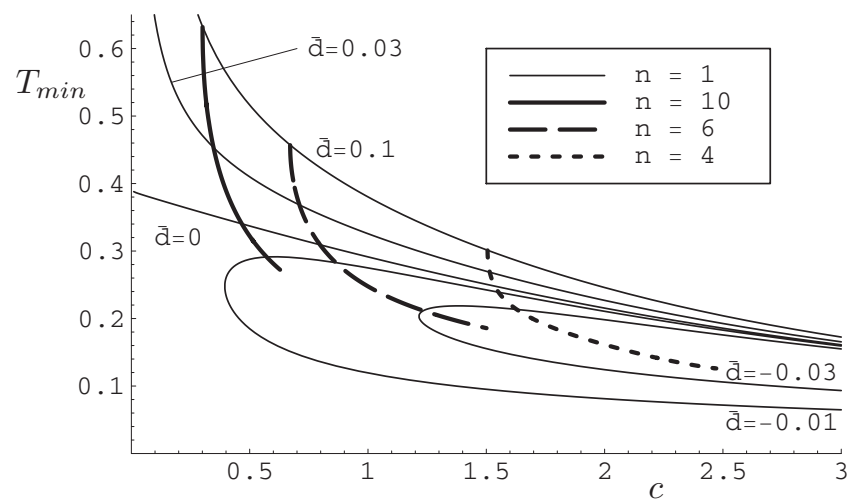

FIG. 1. The dependence of minimal $T$ on dimensionless energy $c$. Thin lines represent the simple periodic solutions with several values of $\bar{d}$ : they correspond, from upper to lower, to $\bar{d}$ $=0.1,0.03,0,-0.01,-0.03$. Thick lines represent multiple period solutions with $\bar{d}=0.1$; the solid line, the line with long dashes, and the line with short dashes correspond, respectively, to $n=10,6$, and 4.

\section{SOLUTIONS WITH MULTIPLE PERIODS}

Next we examine more complex solutions. The TM system determines a z-dependent vector field which maps the $T M$ plane onto itself. Let us call this transformation $g^{z}$. Then $g \equiv g^{1}$ is a mapping performed by one section of a communication line. A simple periodic solution corresponds to a point mapped onto itself: $T^{*}, M^{*}: g^{1}\left(\left\{T^{*}, M^{*}\right\}\right)=\left\{T^{*}, M^{*}\right\}$. In other words, the point $\left\{T^{*}, M^{*}\right\}$ is a stable point of the mapping $g$. In its vicinity, $g$ can be expanded in series with respect to $\delta T=T-T^{*}, \delta M=M-M^{*}$. In the area where this series converge, the motion under $g^{n}$ is regular; outside of the area of convergence, the motion becomes chaotic. The trajectories in the regular area are closed curves, encircling $\left\{T^{*}, M^{*}\right\}$. As it is typical for this mechanism of chaotization, this area is bordered by a separatrix, separating the area where the orbits split into $n$ closed curves, encircling some new central points $\left\{T_{m}^{*}, M_{m}^{*}\right\}$. The outmost $n$ curves form the separatrices, bordering this area on both inner and outer sides. Outside of it there is another area with closed orbits, encircling $\left\{T^{*}, M^{*}\right\}$, then another area of the second type and so on. The structure of the area, separated by each of $n$ outmost curves is the same that of the big area. The $n$ points $\left\{T_{m}^{*}, M_{m}^{*}\right\}$ are (together with $\left\{T^{*}, M^{*}\right\}$ ) the stable points of $g^{n}$. The typical appearance of the trajectories is shown in Fig. 2.

The solutions of $T M$ corresponding to $\left\{T_{m}^{*}, M_{m}^{*}\right\}$ can be found numerically. Figure 3 shows evolution of the $T(z)$ and $M(z)$ for a single period DM soliton and for the four-period solution of $T M$ equations. The energy dependence of $T_{\min }$ of these solutions for $n=10,6$, and 4 at $\bar{d}=0.1$ compared to analogical dependence of the simple periodic solution is shown in Fig. 1. This figure shows that at the same energy the FWHM width is almost two times less than the width of usual DM solitons.

It is customary to describe the properties of DM solitons in terms of map strength and normalized peak power $N_{\text {norm }}^{2}$ $=\sigma P_{0} T_{\mathrm{FWHM}}^{2} /\left(3.11\left|\beta_{2}\right|\right)$ (see Ref. [24]). The dependence of

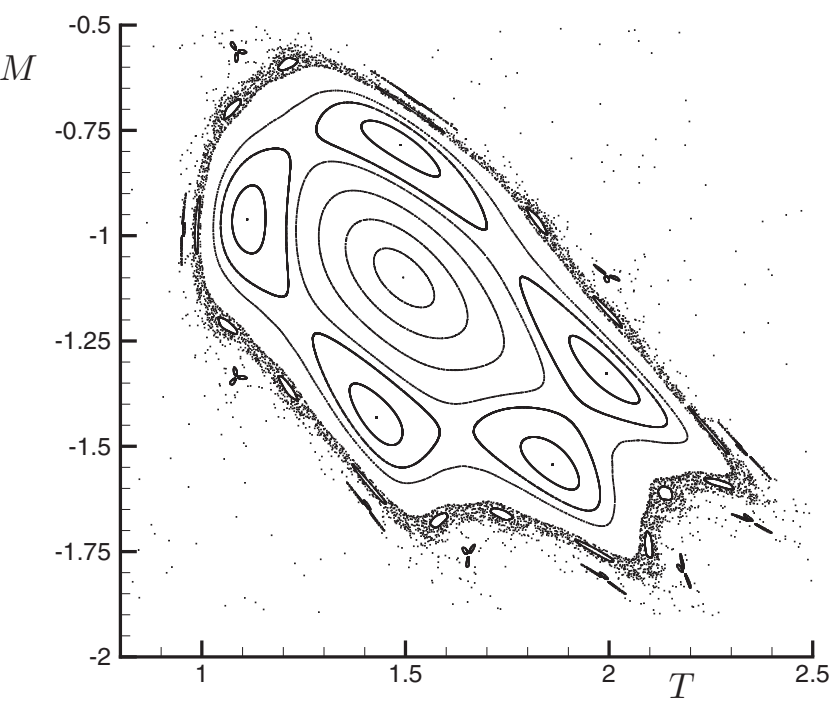

FIG. 2. The trajectories of the points on the $(T, M)$ plane under $g^{m}$ for $c=3.5, \bar{d}=0$.

normalized peak power of multiple period solutions on the map strength (compared to the same of simple period solutions) is presented in Fig. 4. This plot demonstrates that the peak power of multiple-period solitons can be about 3 times less compared to power of conventional DM solitons at the same map strength. The multiple periodic lines branch off the lines corresponding simple periodic solutions on the same map. The line characterizing a multiple period solution disappears when the corresponding point on the phase plane deepens into the chaotic area. As it could be seen, the solutions with lower $n$ appear at higher energies. For zero aver-
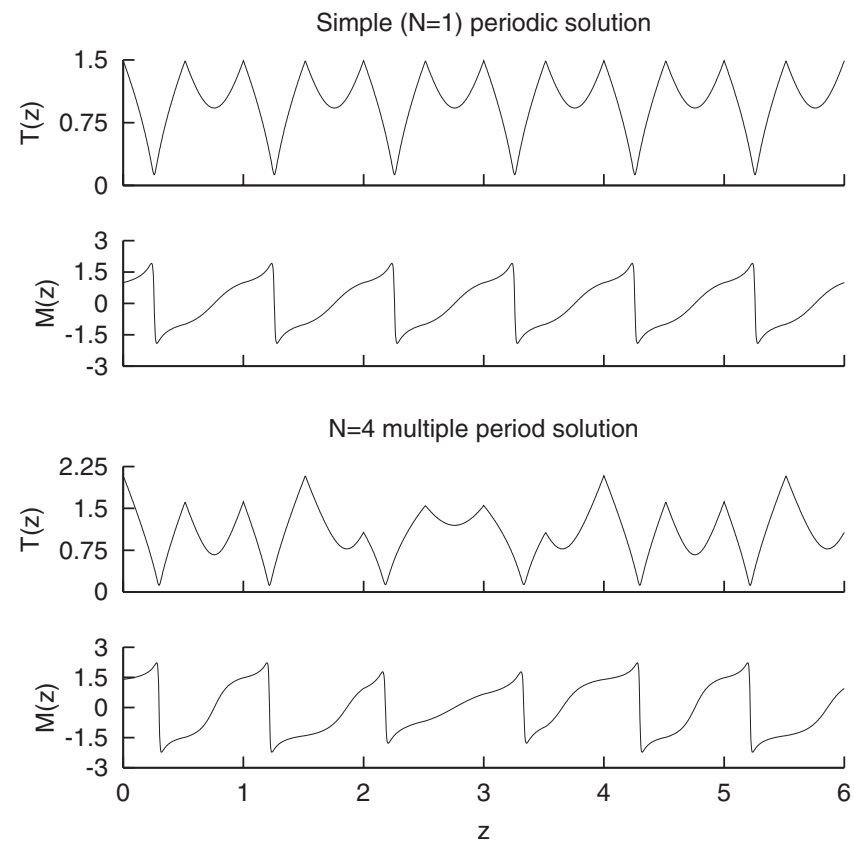

FIG. 3. Evolution of $T$ and $M$ for a single period DM soliton (the upper two plots) and four-period solution (the lower two plots). Here $c=3.94, \bar{d}=0.03$, and $d_{1}=\left|d_{2}\right|=L=1$. 

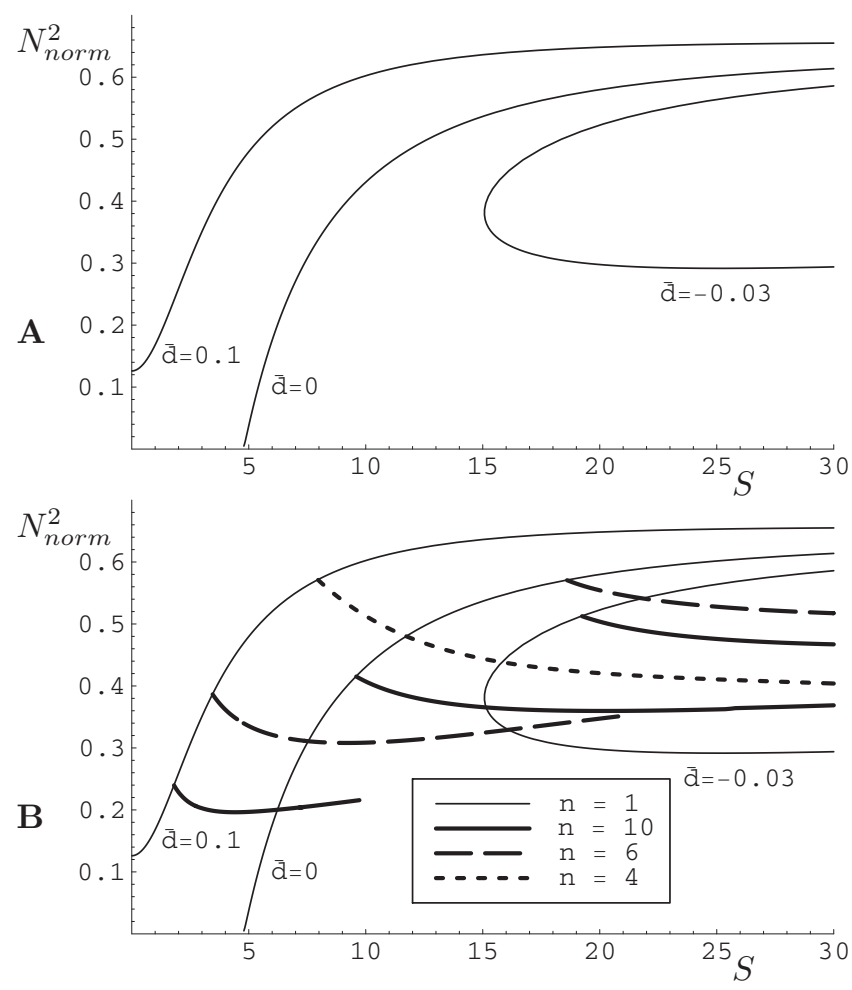

FIG. 4. Dependence of the normalized average power $N_{\text {norm }}^{2}$ on map strength $S$. The plot $A$ represents the simple periodic solutions with $\bar{d}=0.1,0,-0.03$. On the plot $B$ the same simple periodic solutions are compared to multiple-period solutions (thick lines). The solid, long-dashed, and short-dashed thick lines correspond, respectively, to $n=10,6$, and 4 .

age dispersion there is an $n$-period solution for every integer $n \geqslant 4$.

It is evident from the structure of the trajectories of the phase plane points under $g^{n}$ (Fig. 2) that in the vicinity of every stable point of $g^{n}$ there can exist the stable points of $\left(g^{n}\right)^{m}$ for some $m$. They could be called the long-period solutions of the second order, or $(n \times m)$-period solutions. For instance, the structure resembling three-leaved flower in Fig. 2 indicate the presence of solutions with $n=11, m=3$. The lines, representing such solutions on a $T_{\min }$ vs $c$ diagram must be starting from a branch of its "mother" $n$-period solutions in the same way the $n$-period branches start from the simple period line. Undoubtedly, for large total periods the solutions of the higher orders can also be found; however, this is beyond the scope of this paper.

\section{MULTIPLE-PERIOD SOLUTIONS OF NLSE}

In this section we briefly discuss a connection between found new solutions of the TM model and the solution of Eq. (1). Exact numerical solutions of Eq. (1) can be found using integral equation approach developed in Refs. $[11,23,26,32-34]$. In the limit $\sigma \rightarrow 0$, Eq. (8) diverges into the linear Schrödinger equation of a harmonic oscillator $(z$ dependence of the coefficient $d / T^{2}$ can always be removed by a redefinition of $z$ ). This means that provided $T(z), M(z)$ are some periodic solutions of (linear) TM equations, then oscillator modes (the Gauss-Hermite functions) are periodic solutions of Eq. (8). In the case of a small nonlinearity the perturbation theory $[19,31]$ can be applied to describe singleand multiple-period DM solitons. There is a direct analogy between the considered problem and a nonlinear macroscopic quantum oscillator. The periodic oscillations of the phase (that occur due to periodic variation of the dispersion) form an effective parabolic trapping potential. In the linear case any propagating wave is a superposition of the eigenfunctions of such an effective quantum oscillator potential. When nonlinearity comes into play, the energy is redistributed between different modes. Applying an orthogonal set of the Gauss-Hermite functions one can quantify the process of energy redistribution between higher-order modes of the harmonic oscillator. Mathematically this leads to representation of the original NLSE as a set (infinite) of ordinary differential equations for the coefficients of expansion in the basis of Gauss-Hermite functions [19,31] with coefficients given by solution of (nonlinear) model (7). We are mostly interested in the periodic (nonlinear Bloch-wave type) solutions with the lowest energy, which are generated by the energy redistribution from the ground state of the linear oscillator. Consideration of the nonlinear TM equations (7) reduces the rate of the energy flow from the ground state. The DM soliton can be treated as a ground state of an effective macroscopic nonlinear quantum oscillator. For solutions of the NLSE constructed as described, a function $T$ in the $T M$ system (7) can be regarded as a measure of the pulse width: for the low nonlinearity it is simply proportional to $T_{\mathrm{FWHM}}$. Energy redistribution between modes changes the coefficient of proportionality. However, basing on the studies performed for a single-period DM soliton, one might expect that the solutions of NLSE (1) corresponding to multiple-period solutions will have smaller minimal width for the same energy, or equivalently, smaller energy for the same width, compared to usual DM solitons similar to solutions described by the TM model.

\section{CONCLUSIONS}

A class of multiple-period solutions of the nonlinear Schrödinger equation with periodic variations of dispersion is examined using reduction to a set of ordinary differential equations for a pulse width and chirp. The multiperiod solutions found have smaller energies compared to single-period traditional DM solitons. Such multiperiod solutions can be of interest as carrier pulses in high-speed optical communication. In particular, they can be used as carrier pulses in systems employing advanced modulation formats with periodic distribution of intensity in time (having signal in each time slot), e.g., differential phase-shift-keying formats. Another practical application of multiple-period DM solitons can be found in laser systems using dispersion management of the laser cavity. In particular, performance of high power fiber lasers critically depend on a proper dispersion mapping of generated pulses. The presented mathematical theory of multiple-period DM solitons is generic and can be applied to a number of physical systems with periodic variations of dispersive and nonlinear characteristics. 
[1] A. C. Newell and J. V. Moloney, Nonlinear Optics (AddisonWesley, Redwood City, CA, 1992).

[2] A. C. Scott, Nonlinear Science: Emergence and Dynamics of Coherent Structures (Oxford Univeristy Press, Oxford, 1999).

[3] M. J. Ablowitz and P. A. Clarkson, Solitons, Nonlinear Evolution Equations, and Inverse Scattering (Cambridge University Press, Cambridge, UK, 1991).

[4] A. Hasegawa and Y. Kodama, Solitons in Optical Communications (Clarendon, Oxford, 1995).

[5] V. E. Zakharov and E. S. Wabnitz, Optical Solitons: Theoretical Challenges and Industrial Perspectives (Springer-Verlag, Heidelberg, 1998).

[6] P.-L. Sulem and C. Sulem, The Nonlinear Schrödinger Equation: Self-focusing and Wave Collapse (Springer, Berlin, 1999).

[7] A. I. Maimistov and A. M. Basharov, Nonlinear Optical Waves (Springer, Berlin, 1999).

[8] G. Agrawal and Y. Kivshar, Optical Solitons: From Fibers to Photonic Crystals (Academic Press, Amsterdam, 2003).

[9] L. F. Mollenauer and J. P. Gordon, Solitons in Optical Fibers: Fundamentals and Applications (Academic, New York, 2006).

[10] F. Dalfovo, S. Giorgini, L. P. Pitaevskii, and S. Stringari, Rev. Mod. Phys. 71, 463 (1999).

[11] S. K. Turitsyn, E. G. Shapiro, S. B. Medvedev, M. P. Fedoruk, and V. K. Mezentsev, C. R. Phys. 4, 145 (2003).

[12] A. Hasegawa, Y. Kodama, and A. Maruta, Opt. Fiber Technol. 3, 197 (1997).

[13] T. Georges and F. Favre in Proceedings of the International Symposium on New Trends in Optical Soliton Transmission Systems New Trends, Kyoto, 1997, edited by A. Hasegava (Springer, New York, 1998).

[14] R.-J. Essiambre, B. Mikkelsen, and G. Raybon, Electron. Lett. 35, 1576 (1999).

[15] P. V. Mamyushev and N. A. Mamysheva, Opt. Lett. 23, 1523 (1999).

[16] J. M. Soto-Crespo, M. Grapinet, P. Grelu, and N. Akhmediev, Phys. Rev. E 70, 066612 (2004).

[17] A. N. Pilipetskii, in Proceedings of Optical Fiber Communi- cation Conference, 2006 and the 2006 National Fiber Optic Engineers Conference, Anaheim, 2006 (IEEE, New York, 2006)

[18] H. A. Haus, K. Tamura, L. E. Nelson, and E. P. Ippen, IEEE J. Quantum Electron. 31, 591 (1995).

[19] S. K. Turitsyn, T. Shafer, K. H. Spatschek, and V. K. Mezentsev, Opt. Commun. 163, 122 (1999).

[20] J. D. Ania-Castanon, T. J. Ellingham, R. Ibbotson, X. Chen, L. Zhang, and S. K. Turitsyn, Phys. Rev. Lett. 96, 023902 (2006).

[21] M. Suzuki, I. Morita, N. Edagawa, S. Yamamoto, H. Taga, and S. Akiba, Electron. Lett. 31, 2027 (1995).

[22] N. Smith, F. M. Knox, N. J. Doran, K. J. Blow, and I. Bennion, Electron. Lett. 32, 54 (1995).

[23] I. Gabitov and S. K. Turitsyn, Opt. Lett. 21, 327 (1996).

[24] A. Bernston, N. J. Doran, W. Forysiak, and J. H. B. Nijhof, Opt. Lett. 23, 900 (1998).

[25] J. Kutz, P. Holmes, S. G. Evangelides, Jr., and J. P. Gordon, J. Opt. Soc. Am. B 15, 87 (1998).

[26] I. Gabitov, E. G. Shapiro, and S. K. Turitsyn, Phys. Rev. E 55, 3624 (1997).

[27] T. I. Lakoba and D. J. Kaup, Electron. Lett. 34, 1124 (1998).

[28] T. I. Lakoba and D. J. Kaup, Phys. Rev. E 58, 6728 (1998).

[29] I. Gabitov and S. K. Turitsyn, JETP Lett. 63, 861 (1996).

[30] S. K. Turitsyn, T. Schaefer, and V. K. Mezentsev, Phys. Rev. E 58, R5264 (1998).

[31] T. I. Lakoba, J. Yang, D. J. Kaup, and B. A. Malomed, Opt. Commun. 149, 366 (1998).

[32] M. J. Ablowitz and G. Biondini, Opt. Lett. 23, 1668 (1998).

[33] P. M. Lushnikov, Opt. Lett. 25, 1144 (2000).

[34] I. Gabitov, R. Indik, L. Mollenauer, M. Shkarayev, M. Stepanov, and P. M. Lushnikov, Opt. Lett. 32, 605 (2007).

[35] For Gaussian pulse $T_{\mathrm{FWHM}} \simeq 1.665 T$.

[36] Standing on the general grounds, one should expect that the system would be characterized by the number of parameters equal to the number of dimensionless parameters that can be constructed from the initial set of values, and that is 4 . However, this system has an extra symmetry transform, which reduces this number to 3 . 